\section{Numerical investigation of breast tumour detection using multi-static radar}

R. Nilavalan, A. Gbedemah, I.J. Craddock, X. Li and S.C. Hagness

\begin{abstract}
A breast cancer detection technique using multi-static radar is proposed. For the first time, images are produced using this technique, using backscatter data produced from an anatomically realistic $2 \mathrm{D}$ MRI-derived FDTD model of the breast. Successful detection of a $2 \mathrm{~mm}$-diameter tumour is demonstrated, although clarity of detection is dependent on mitigating antenna mutual coupling and skin reflections.
\end{abstract}

Introduction: Breast cancer is one of the most common cancers in women. In 2003, in the US alone, it is estimated that a woman will be diagnosed with breast cancer every three minutes [1]. X-ray mammography is currently the most effective detection technique [2], however it suffers from relatively high missed- and false-detection rates and involves uncomfortable compression of the breast. X-rays are also ionising and this poses limitations on the frequency of screening.

Microwave detection of breast tumours is a non-ionising, potentially low-cost modality that relies on the dielectric contrast between healthy and malignant breast tissues [3, 4]. The work presented in this Letter falls under the class of ultra-wideband microwave radar techniques, which take advantage of the relatively large microwave scattering crosssections of malignant tumours [5]. Previous theoretical investigations of such techniques have focused on monostatic system configurations [5-8]. This contribution presents results from a hitherto unexplored variant, based on a multi-static radar technique originally developed for use in landmine detection [9].

Proposed technique: multistatic radar: The problem of detecting buried antipersonnel landmines bears considerable likeness to the detection of breast tumours. In both cases the objective is to detect the presence of a compact object with dielectric properties distinct from the surrounding lossy, inhomogeneous medium. This is problematic due to the trade-off between resolution and depth (a result of the increasing dielectric losses with frequency) and the presence of clutter (unwanted reflection from the medium).

The technique employed to counter clutter in [9] may be briefly described as follows. In Fig. 1, an array of $N$ antennas is close to, or in contact with the medium of interest. Each antenna in turn transmits a pulse and a single time-shared detector records the received signal $y_{i}(t)$ at each of the other antennas. Since monostatic operation is excluded and as interchanging transmit and receive antennas would not produce any additional information, the total number of transmissions recorded is $N(N-1) / 2$.

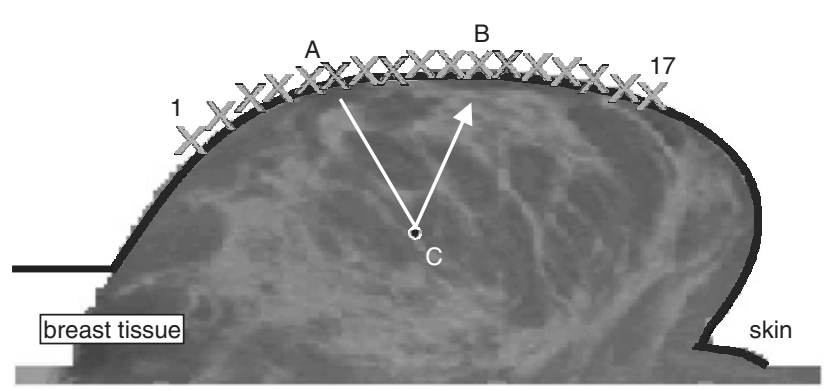

Fig. 1 Transmit antenna $A$, receive antenna $B$, point of interest $C$

The recorded data is then synthetically focused at any point of interest in the volume beneath this ground by time-aligning the signals $y_{i}(t)$, using the estimated propagation time $T_{i}$ from the transmit antenna $\mathrm{A}$ to the receive antenna $\mathrm{B}$ via the point of interest $\mathrm{C}$. The return from $\mathrm{C}$ is then computed by integrating the data over a window corresponding to the transmit pulse width $\tau$.

$$
V=\int_{0}^{\tau}\left(\sum_{i=1}^{N(N-1) / 2} w_{i} y_{i}\left(t-T_{i}\right)\right)^{2} d t
$$

where $w_{i}$ are factors that are applied to compensate for differences in the predicted attenuation between the round-trip paths (depending on the depth of point $\mathrm{C}$ and the spacing from $\mathrm{A}$ to $\mathrm{B}$ ).

The signal processing approach of (1) is similar to other time-shiftand-sum beamforming algorithms [5-7] but differs from them in its use of all possible transmit/receive combinations in the array. In an operational system the increased number of observations may be exploited to offer additional clutter rejection. We note that other clutter-suppression options such as least-squares optimal beamforming [8] have not been explored here.

A validation exercise, using modelled data, is described in the following Section.

Test model: A 2D FDTD model was used to simulate an antenna array placed along the surface of a naturally flattened breast of a patient in a supine position. The model was generated from MRI data using the procedure described in [7]. Tissue dielectric properties are assumed to be frequency-independent; normal breast tissue heterogeneity is assigned average values of $\varepsilon_{r}=9.8$ and $\sigma=0.4 \mathrm{~S} / \mathrm{m}$ with a $\pm 10 \%$ variation about the average and the properties of the $2 \mathrm{~mm}$-diameter malignant tumour are assumed to be $\varepsilon_{r}=50$ and $\sigma=7 \mathrm{~S} / \mathrm{m}$. A $2 \mathrm{~mm}$ thick layer of skin $\left(\varepsilon_{r}=36\right.$ and $\left.\sigma=4 \mathrm{~S} / \mathrm{m}\right)$ is included. An immersion medium with dielectric properties matching average normal breast tissue is assumed to be present above the antenna array and breast in the simulations.

In the 2D model, the antenna array consists of 17 hard sources, equally spaced and spanning $8 \mathrm{~cm}$ in the lateral direction. In each simulation, one element in the antenna array is excited with a short pulse. Magnetic fields (proportional to induced current) are observed and recorded at all antenna locations.

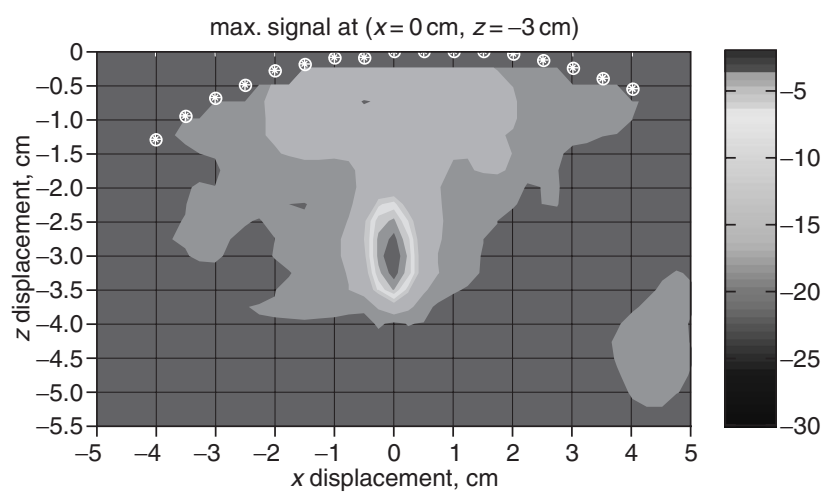

Fig. 2 Breast image reconstructed from backscatter waveforms, after mutual couplings and skin reflections removed via calibration Tumour at $x=0, z=-3 \mathrm{~cm}$

Results: Figs. 2 and 3 demonstrate the results of applying (1) to backscatter data from the FDTD simulations. Mutual couplings between the antennas, and the reflection from the skin-breast interface must be reduced as much as possible. One simple technique that could be used in practice would be to calibrate the array by recording the couplings with the array in contact with a uniform skin/breast phantom. Fig. 2 shows the results obtained using this technique (using calibration data from an FDTD model incorporating a homogeneous breast). In this image, the $2 \mathrm{~mm}$ tumour is clearly visible at the correct location, with a signal strength $18 \mathrm{~dB}$ above the highest response from the surrounding clutter.

While a calibration of this sort should be possible in practice, its success will be to some extent patient-specific. One alternative is to employ windowing techniques to eliminate the unwanted signals. In this case, the time delays for the unwanted signals are computed and the corresponding signals are neglected in the focusing process without any calibration or subtraction. The results (Fig. 3) are noticeably degraded compared with Fig. 2; however the tumour is still clearly identifiable and stands at least $6 \mathrm{~dB}$ above the surrounding clutter. An alternative not explored here involves the use of a data-adaptive algorithm to estimate the undesired artifact in each channel using the backscatter waveforms [8]. 


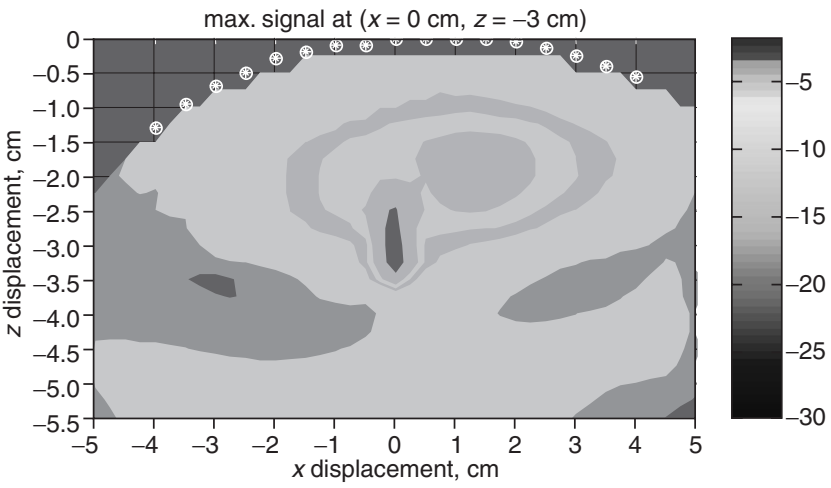

Fig. 3 Breast image reconstructed from backscatter waveforms, after mutual couplings and skin reflections removed using windowing Tumour at $x=0, z=-3 \mathrm{~cm}$

Conclusions: Initial results have been presented using a multi-static radar approach to imaging of the human breast. These results, using data from a realistic FDTD model, demonstrate successful detection of a small tumour in a lossy, inhomogeneous human breast. The radar returns from the inhomogeneous structure of the breast do not significantly mask the tumour, even if an initial calibration of the array is not possible. Future work includes the development of 3D FDTD models and an experimental system employing an array of wideband patch antennas, a vector network analyser, and a tissue equivalent phantom.

(C) IEE 2003

Electronics Letters Online No: 20031183

9 September 2003 DOI: 10.1049/el:20031183
R. Nilavalan, A. Gbedemah and I.J. Craddock (Centre for Communications Research, University of Bristol, Merchant Venturers Building, Woodland Road, Bristol BS8 1UB, United Kingdom)

X. Li and S.C. Hagness (Department of Electrical and Computer Engineering, University of Wisconsin-Madison, 1415 Engineering Drive, Madison, WI 53706, USA)

\section{References}

1 'Cancer facts and figures 2003', American Cancer Society, www.cancer.org

2 BROWN, M., et al.: 'Screening mammography in community practice', Amer. J. Roentgen., 1995, 165, pp. 1373-1377

3 JOINES, W.T., DHENXING, Y.Z., and JIRTLE, R.L.: 'The measured electrical properties of normal and malignant human tissues from 50 to $900 \mathrm{MHz}$ ', Med. Phys., 1994, 21, pp. 547-550

4 CHAUDHARY, S.S., et al.: 'Dielectric properties of normal and malignant human breast tissues at radiowave and microwave frequencies', Indian $J$. Biochem. Biophys., 1984, 21, pp. 76-79

5 HAGNESS, S.C., TAFOVE, A., and BRIDGES, J.E.: 'Two-dimensional FDTD analysis of a pulsed microwave confocal system for breast cancer detection: fixed-focus and antenna-array sensors', IEEE Trans. Biomed. Eng., 1998, 45, (12), pp. 1470-1479

6 FEAR, E.C., and STUCHLY, M.A.: 'Microwave detection of breast cancer', IEEE Trans. Microw. Theory Tech., 2000, 48, (11), pp. 1854-1863

7 LI, X., and HAGNESS, S.C.: 'A confocal microwave imaging algorithm for breast cancer detection', IEEE Microw. Wirel. Compon. Lett., 2001, 11, pp. $130-132$

8 BOND, E.J., et al.: 'Microwave imaging via space-time beamforming for early detection of breast cancer', IEEE Trans. Antennas Propag., 2003, 51, (8), pp. 1690-1705

9 BENJAMIN, R., et al.: 'Microwave detection of buried mines using noncontact, synthetic near-field focusing', IEE Proc., Radar Sonar Navig., 2001, 148, (4), pp. 233-240 